DE DE GRUYTER

OPEN

G

BULGARIAN ACADEMY OF SCIENCES

CYBERNETICS AND INFORMATION TECHNOLOGIES • Volume 15, No 5

Special Issue on Control in Transportation Systems

Sofia • 2015

Print ISSN: 1311-9702; Online ISSN: 1314-4081

DOI: 10.1515/cait-2015-0017

\title{
Speed Profile Prediction in Intelligent Transport Systems Exemplified by Vehicle to Vehicle Interactions
}

\author{
Ivan Bosankic, Lejla Banjanovic-Mehmedovic, Fahrudin Mehmedovic \\ IVL, Tuzla, Bosnia and Herzegovina, Faculty of Electrical Engineering, University of Tuzla, Bosnia \\ and Herzegovina, ABB Representative Tuzla, Bosnia and Herzegovina \\ Emails:ivan.bosankic@ivl.ba lejla.mehmedovic@untz.ba fahrudin.mehmedovic@hr.abb.com
}

\begin{abstract}
Intelligent Transport Systems (ITS) fall in the framework of cyberphysical systems due to the interaction between physical systems (vehicles) and distributed information acquisition and dissemination infrastructure. With the accelerated development of wireless Vehicle-to-Vehicle $(\mathrm{V} 2 \mathrm{~V})$ and Vehicle-to Infrastructure (V2I) communications, the integrated acquiring and processing of information is becoming feasible at an increasingly large scale. Accurate prediction of the traffic information in real time, such as the speed, flow, density has important applications in many areas of Intelligent Transport systems. It is a challenging problem due to the dynamic changes of the traffic states caused by many uncertain factors along a travelling route. In this paper we present a V2V based Speed Profile Prediction approach (V2VSPP) that was developed using neural network learning to predict the speed of selected agents based on the received signal strength values of communications between pairs of vehicles. The V2VSPP was trained and evaluated by using traffic data provided by the Australian Centre for Field Robotics. It contains vehicle state information, vehicle-to-vehicle communications and road maps with high temporal resolution for large numbers of interacting vehicles over a long time period. The experimental results show that the proposed approach (V2VSPP) has the capability of providing accurate predictions of speed profiles in multi-vehicle trajectories setup.
\end{abstract}

Keywords: Cyber-Physical systems, Intelligent Transport systems, Vehicle-toVehicle interaction $(\mathrm{V} 2 \mathrm{~V})$, neural networks, speed profile prediction, traffic data.

\section{Introduction}

Cyber-Physical Systems (CPS) are emerging as an integrative research field aimed towards a new generation of engineered systems [22]. Cyber-Physical Systems 
integrate computing and communication capabilities by monitoring and controlling the physical systems via embedded hardware and computers [20]. CPSs are mostly Distributed Parameter Systems (DPSs) and dynamic evolutions happen not only along the time axis but also along spatial axes.

Today, CPS can be found in such diverse industries as aerospace, automotive, energy, healthcare, manufacturing, infrastructure, consumer electronics and communications. Building efficient CPSs of the future requires multi-disciplinary skills. In particular, the confluence of real-time computing, embedded systems, wireless sensor networks, control theory, signal processing and knowledge creation using artificial intelligence are required to create these new systems.

Intelligent Transport Systems (ITS) fall in the framework of cyber-physical systems due to the interaction between physical systems (vehicles) and distributed information acquisition and dissemination infrastructure (wireless networks, sensors, actuators, processors and accompanying software). Networking in cyberphysical systems creates new ways of avoiding accidents, respecting limited energy resources and reducing environmental pollution. Wireless ad hoc communication has been identified as a major component to enable cooperative safety and automation by major vehicle manufacturers. Heterogeneous networking: OBD [11] and CAN-Bus [2] have been developed for intra-vehicle communications. DSRC/WAVE [5] are defined for V2V and V2R communications.

One of the cornerstones of ITS is the ability of vehicles and infrastructure to interact in meaningful ways to improve the safety and efficiency of the system. There are many applications in ITS which are being developed based upon vehicle to vehicle, and vehicle to infrastructure communication [28]. Wireless communication proposed in the applications involves sharing of perception data [17], position information [2], vehicle tracking [30], collision avoidance [29, 3], controller design [9] and cooperative driving challenges [14, 23]. Through intervehicle communications, rear-end collision prevention is discussed in [1, 33].

Accurate prediction of the traffic information is important in many applications of Intelligent Transport Systems in order to reduce the uncertainty of future traffic states, improve traffic mobility, providing the driver with realistic estimation of the travel times and expected delays, and alternative routes to destinations. The research of others showed that an accurately predicted vehicle speed profile of an intended route is important to achieve optimal fuel economy $[6,12]$ and estimated distance [19].

There is much research being conducted about the behavior of drivers and techniques to predict this behavior [21, 8]. The Advanced Driver Assistance Systems (ADAS) are becoming increasingly popular in commercially available vehicles. There is also a great deal of interest in exploring the driver behavior in the area of ITS towards creating autonomous vehicles [28]. Deployment and adoption of these vehicles will require autonomous systems to interact with human drivers. The techniques for understanding and predicting driver's behaviour are necessary for autonomous vehicles to be successful.

In this paper we present the V2V based Speed Profile Prediction approach (V2VSPP) that was developed using neural network learning. There is also a great 
deal of interest in exploring driver's behaviour in the area of Intelligent Transportation Systems to predict the travelling speed profile for selected agents on the travelling routes. The V2VSPP was trained and evaluated by using traffic data provided by the Australian Centre for Field Robotics, which is introduced in [28]. It contains vehicle state information, vehicle-to-vehicle communications and road maps with high temporal resolution for large numbers of interacting vehicles over a long time period. The forecasting is made based on the distance between vehicles, the received signal strength values for communications between pairs of vehicles. The V2VSPP can predict the traffic speed profile for each vehicle agent. The experimental results show that the proposed approach (V2VSPP) has the ability of providing accurate predictions of the speed profiles in multi-vehicle trajectories setup.

\section{Intelligent transportation systems}

Transportation systems consist of embedded control systems inside the vehicle and the surrounding infrastructure, as well as the interaction between the vehicles and the infrastructure [15]. In the field of mobility, i.e., transportation, an extensive networking of the different means of transportation is possible only if using cyberphysical systems.

The Intelligent Transportation Systems (ITS) have identified a number of key technological requirements for cyber-physical systems [16]:

- Wireless communications. The wireless communication has become a large area. Wireless networks connect the devices, transmit data through signals and use any medium (radio wave, microwave) for transferring and sharing the data between nodes. Wireless communication has various sub domains, such as ad hoc networks, sensor networks, mesh networks, cellular networks, which are different in nature in terms of packet types, resources and infrastructure. WiFi/ZigBee/IEEE 802.15.4 is commonly assumed in such systems.

- CPS networking can stem from hierarchically interconnected networks, mostly Internet, local area wired and wireless networks, and wireless sensor networks. Internet access to individual components of distributed embedded systems can be based on both wired and wireless LAN technologies, predominantly on IEEE 802.3 and related Ethernet standards, and on IEEE 802.11 WiFi and associated wireless LAN protocols. Particular embedded systems and their components can be attached directly to Ethernet with the help of TCP/IP protocol stack, but also indirectly or exclusively through various wired Fieldbuses or wireless technologies, such as IEEE 802.11b and IEEE 802.15.4 with related ZigBee.

- Miniature hardware (nano-technology) can concurrently optimize both hardware (nm CMOS) and embedded software. Example: System on Chip (FPGA).

- Computational Technologies. With the development of technologies, computational science is used in different fields. In intelligent transportation system the computational technologies provide a platform and development in architecture and software for real time applications. This type of a platform includes model- 
based process control, ubiquities computing and artificial intelligence [18]. The application of ITS has many real time operating systems, rich microprocessors, memories and hardware installed in vehicles.

- Distributed IT architectures for ITS. Service-Oriented Architectures (SOA) refer to a new paradigm for building reliable distributed systems, where the functions are composed as services and all the interacting components are loosely coupled. Web services are used to implement software components in SOA [10]. On the other hand, cloud computing has become cost-effective and popular technology for using distributed resources efficiently as it allows to bind them together to process a large volume of data or to solve large scale problems [25]. Grid computing is another popular approach for accessing geographically distributed resources, such as computer, storage systems, data sources, services, equipments, etc. Grid computing utilizes the resources of multiple computers to solve a particular problem cooperatively, without tight coupling. In grid computing, a large problem is divided among several workstations in order to ensure the best use of available resources in a cost-efficient way. Researches of the last few years have shown that the aims of cloud and grid computing are overlapping with the goals of service oriented architectures based on web services. Therefore, it is natural to apply distributed systems technology to solve the problems of ITS.

- Sensing Technologies. Sensing Technology in a transportation system is inevitable technology. The Wireless Sensor Networks (WSNs) have a large number of sensor nodes representing significant efficiency over traditional sensors [31]. Sensor technologies are designed for dissimilar scenarios in intelligent transportation system applications, for instance traffic condition monitoring. These applications run in real time; therefore, end-to-end delay and synchronization is critical for such systems [27]. In WSN the sensors or nodes, which are deployed for data gathering and one or more sink nodes are connected through different long range connections, i.e., satellite, WiFi, etc., [24]. Each autonomic node in a sensor network is equipped with: a radio transceiver or another wireless communication device, a processing unit which can be a small micro-controller, a sensing unit and an energy source and a mobilizer is needed to move a sensor node from the current position and carry out assigned tasks.

ITSs are important applications of CPS. Methods and tools of artificial intelligence, which are used primarily in Intelligent Transport Systems, are: behavior predicting of the transportation systems, transportation optimization problems in control systems, clustering, planning process, decision making and pattern recognition.

\section{V2V based speed profile prediction using neural learning}

Intelligent Transportation Systems rely on understanding, predicting and affecting the interactions between vehicles. Development of such systems must be based on the data derived from the actual wireless Vehicle-to-Vehicle (V2V) and Vehicle-toInfrastructure (V2I) communications in real world applications. Understanding of 
how these interactions affect, for example the speed profile of vehicles, is also necessary to create robust systems.

The speed profile can be estimated from historical data through analyzing limited traffic information from fields. For instance, the traffic data, such as distance, location, etc. from a vehicle (agent) could be linked with historical travel speed data. The various techniques, e.g., parametric models (neural networks, wavelet), semi-parametric models (Fuzzy theory, neuro-fuzzy), statistics and mathematical models, could be adopted to develop a parameter estimation model [26]. The research studies have displayed that the combination between historical data and estimation theory could provide well estimation capacity with a fluency traffic flow condition. However, for congested and changeful environment, a hybrid method might be needed for improving the accuracy of the estimation method.

The predicted (or approximated) model of the output signal is presented by

$$
\hat{y}=f(\boldsymbol{m}(k), p)=\sum_{i=1}^{M} p_{i} \kappa\left(\boldsymbol{m}(k), \beta_{i}, \gamma_{i}\right),
$$

which can be considered as a nonlinear mapping from past data, representing the regressors, i.e., the elements of $\boldsymbol{m}(k)$, with the dilation parameters $\beta_{i}$ and translation parameters $\gamma_{i}$. Usually we are interested in the multi-variable case when $\boldsymbol{m}(k)$ consists of more than one element. Any multivariable function $f_{0}[$.] can be approximated reasonably accurately by $f$ [.] according to the above equation by selecting an appropriate $\kappa[$.] for a sufficiently large value of $M$. As the basis functions usually contain adjustable parameters, these model structures can also be considered as basis function network models. They are characterized by one mother basic function which defines the nonlinear network models, such as Artificial Neural Network (ANN) models, wavelet models or even classical nonlinear blackbox models [26] .

\subsection{Artificial neural networks}

Artificial Neural Networks (ANN), as universal approximates, are capable of modeling complex mappings between the inputs and outputs of a system up to an arbitrary precision. On the other hand, another important property of the neural network is its ability to learn complex nonlinear relationships between the inputs and outputs of the network [32]. Learning is a process, through which the implicit rules are extracted from the patterns of experience.

Traffic information predictions, such as speed, flow and travel time are complex nonlinear spatial-temporal problems, for which the dynamics in freeflowing or congested conditions are different. The learning capabilities of Neural Networks (NN) make them a suitable approach for solving the complicated nonlinear traffic prediction problem. The NN-based approaches are relatively less sensitive to erroneous or missing data and they are independent of the particular geometry of the prediction location. The main limitations of NN approaches are the static NN architecture, which may not capture the true dynamics of the real world traffic states. The neural network based approaches also require time-intensive training to learn from traffic training data [32].

Many prediction profiles projects in ITSs have been developed. In [7] the neural network prediction of the spot speed for the lost link's speed based on 
neighboring links' speed is proposed. The time-lag recurrent network to predict the spot speed and travel time at four sensor locations for up to 15 min prediction is presented in paper [4]. In paper [13] an Intelligent Traffic Modeling System (ITMS) was developed to predict the speed profile from the origin to the destination of a given route.

Two of the most used feed forward networks are the MultiLayer Perceptron Networks (MLP) and the Radial Basis Function network (RBF), which is a special case of the Hyper Basis Function networks (HBF) [26]. In MLP Neural Network, there are one or more hidden layers, whose nodes are correspondingly called hidden neurons. The back-propagation learning algorithm network typically trains the network by employing the deviation of the outputs from the corresponding desired values to correct and update the weights of the previous layer. However, a more accurate result can be achieved by using methods like Levenberg-Marquardt learning algorithm [32].

The concept of RBF neural networks is closely related to MLP-networks. However, RBF networks in contrast to MLP-networks were not biologically inspired, but rather originated from the interpolation of multivariable functions. Usually, the structure consists of an input layer for L0 inputs, one hidden layer with L1 neurons and an output layer consisting of L2 neurons corresponding to the number of model outputs.

Herein the application of ANN architectures as an approximation tool for predicting the speed profile of $\mathrm{V} 2 \mathrm{~V}$ based interactions performance is assessed, including Multi-Layer Feed forward Networks (or Multi-Layer Perceptron (MLP)). In a MLPNeural Network there are one or more hidden layers, whose nodes are correspondingly called hidden neurons. The back-propagation learning algorithm network typically trains the network by employing the deviation of the outputs from the corresponding desired values to correct and update the weights of the previous layer. However, a more accurate result can be achieved by using methods as Levenberg-Marquardt learning algorithm [31].

The proposed methodology used to develop the V2VSPP is different from the published technologies in the following major aspects:

- We addressed the problem by developing NNs for learning different vehicle traffic conditions for the received communication signal between the pairs of vehicles in order to predict the traffic speed.

- The V2SPP was trained and validated on large amounts of real world traffic data.

\subsection{Data set}

In order to introduce new cooperative approaches and algorithms in ITS, it is necessary to have a rich dataset to experiment with. We used a detailed Warrigal dataset, presented in paper [28].This rich dataset, derived from the interactions of large trucks and smaller $4 \mathrm{WD}$ vehicles in an industrial setting, was collected by a fleet of 13 vehicles operating in a large quarry-type environment during a period of 3 years. The dataset includes information about the vehicles' state (e.g., position, speed and heading), as well as details of their peer-to-peer radio communications. 
The data span is within a period of 3 years with a resolution of $1 \mathrm{~Hz}$. It contains vehicle state information, vehicle-to-vehicle communications and road maps of high temporal resolution for large numbers of interacting vehicles over a long time period. Due to its size, the dataset is divided into daily periods.

Peer-to-peer communication between vehicles is effected in two frequencies to improve the redundancy through the complementary properties of each frequency. All vehicles communicate at $2.4 \mathrm{GHz}$ and $433 \mathrm{MHz}$. The light vehicles are fitted with a single $2.4 \mathrm{GHz}$ antenna and a single $433 \mathrm{MHz}$ antenna. The heavy vehicles are fitted with a single $2.4 \mathrm{GHz}$ antenna at the front of the vehicle and a pair of 433 $\mathrm{MHz}$ antennas, one mounted at the front and the other at the rear. The overall structure of the multi-vehicle system is presented in Fig. 1.

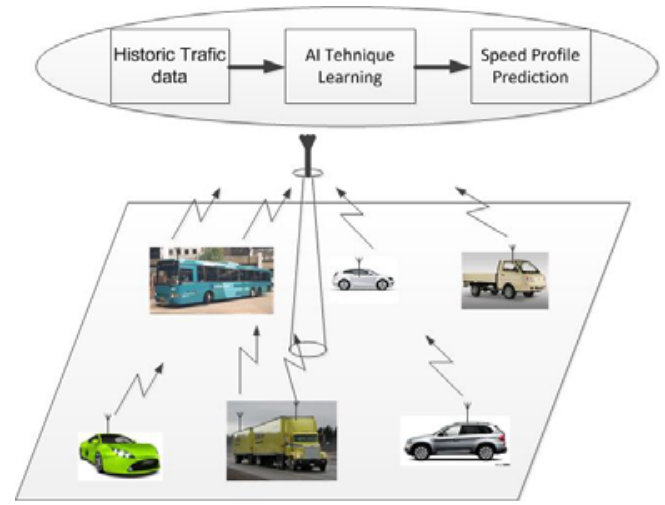

Fig. 1. The overall structure of the multi-vehicle system

Any artificial neural network requires to be trained with adequate data, if it is to provide accurate prediction of the desired outputs from the given inputs. In this case the inputs are the distances between vehicles, the received signal strength for communications at $433 \mathrm{MHz}$ frequency between pairs of vehicles for a particular day. The V2VSPP can predict the traffic speed profile for each vehicle agent. The statistical parameters for the data sets are calculated and given in Tables 1-3.

Table 1. Statistical parameters of the distance data set for different vehicle agents

\begin{tabular}{|c|c|c|c|c|}
\hline \multirow{2}{*}{$\begin{array}{c}\text { Distance } \\
(\mathrm{m})\end{array}$} & \multirow{2}{*}{ Date } & \multicolumn{3}{|c|}{ Agent } \\
\hline & & 52 & 38 & 74 \\
\hline \multirow{3}{*}{ Min } & 2014-03-07 & \multirow{3}{*}{4,23} & \multirow{3}{*}{0} & \multirow{3}{*}{0} \\
\hline & 2014-04-26 & & & \\
\hline & 2014-05-23 & & & \\
\hline \multirow{3}{*}{ Max } & 2014-03-07 & \multirow{3}{*}{3425} & \multirow{3}{*}{3310} & \multirow{3}{*}{4421} \\
\hline & 2014-04-26 & & & \\
\hline & 2014-05-23 & & & \\
\hline \multirow{3}{*}{ Var } & 2014-03-07 & \multirow{3}{*}{28074} & \multirow{3}{*}{15933} & \multirow{3}{*}{14995} \\
\hline & 2014-04-26 & & & \\
\hline & 2014-05-23 & & & \\
\hline \multirow{3}{*}{ Mean } & 2014-03-07 & \multirow{3}{*}{106} & \multirow{3}{*}{67.84} & \multirow{3}{*}{82.41} \\
\hline & 2014-04-26 & & & \\
\hline & 2014-05-23 & & & \\
\hline
\end{tabular}


Table 2. Statistical parameters of the signal strength data set for different vehicle agents

\begin{tabular}{|c|c|r|r|r|}
\hline \multirow{2}{*}{ Signal strength } & \multirow{2}{*}{ Date } & \multicolumn{3}{|c|}{ Agent } \\
\hline \multirow{3}{*}{ Min } & $2014-03-07$ & 38 & \multicolumn{1}{c|}{38} & 74 \\
\cline { 2 - 5 } & $2014-04-26$ & 24 & 46 & 31 \\
\cline { 2 - 5 } & $2014-05-23$ & 37 & 130 & 47 \\
\hline \multirow{3}{*}{ Max } & $2014-03-07$ & 335 & 353 & 336 \\
\cline { 2 - 5 } & $2014-04-26$ & 342 & 347 & 335 \\
\cline { 2 - 5 } & $2014-05-23$ & 335 & 335 & 328 \\
\hline \multirow{3}{*}{ Var } & $2014-03-07$ & 3956 & 3407 & 3665 \\
\cline { 2 - 5 } & $2014-04-26$ & 3702 & 2484 & 2947 \\
\cline { 2 - 5 } Mean & $2014-05-23$ & 3151 & 1574 & 3074 \\
& $2014-03-07$ & 227 & 233 & 220 \\
\cline { 2 - 5 } & $2014-04-26$ & 228 & 239 & 232 \\
\cline { 2 - 5 } & $2014-05-23$ & 239 & 266 & 238 \\
\hline
\end{tabular}

Table 3. Statistical parameters of the velocity data set for different vehicle agents

\begin{tabular}{|c|c|c|c|c|}
\hline \multirow{2}{*}{ Velocity } & \multirow{2}{*}{ Date } & \multicolumn{3}{|c|}{ Agent } \\
\cline { 2 - 5 } & $2014-03-07$ & 52 & 38 & 74 \\
\hline \multirow{3}{*}{ Min } & $2014-04-26$ & 0 & 0 & 0 \\
\cline { 2 - 5 } & $2014-05-23$ & 0 & 0 & 0 \\
\cline { 2 - 5 } & $2014-03-07$ & 0 & 0 & 0 \\
\hline \multirow{3}{*}{ Max } & $2014-04-26$ & 11.9 & 11.8 & 11.8 \\
\cline { 2 - 5 } & $2014-05-23$ & 12.10 & 12.2 & 11.9 \\
\hline \multirow{3}{*}{ Var } & $2014-03-07$ & 13.66 & 11.04 & 11.5 \\
\cline { 2 - 5 } & $2014-04-26$ & 7.44 & 5.80 & 7.00 \\
\cline { 2 - 5 } Mean & $2014-05-23$ & 18.80 & 6.21 & 12.58 \\
\cline { 2 - 5 } & $2014-03-07$ & 2.31 & 2.36 & 1.43 \\
\hline & $2014-04-26$ & 1.21 & 0.85 & 1.13 \\
\hline
\end{tabular}

\section{Experiments and approach evaluation}

In this paper the MLP and RBF neural networks prediction was developed using the Matlab/NN toolbox in order to earn the same training data set. The training sessions for the ANN structures (for each vehicle agent) were performed by using the data for a selected vehicle agent from the sample pool. The performance and robustness of the different networks were compared, so that the best configuration in terms of accuracy, performance and cost could be selected among the available architectures.

Three random dates from the Warrigal dataset were selected: 2009-03-07, 2009-04-26 and 2009-03-23. For these dates, three agents and their interactions were considered and analyzed: agents 52,38 and 74 . The specified date routes for three agents are illustrated in Figs 2, 3 and 4.

To achieve speed estimation, our neural network learned mapping from the combination of the signal strength and the distance between two vehicles to vehicle (agent) speed. The received signal strength data was divided in 7 equally distributed clusters labeled 1-7, based on the intensity of the signal. The set of input data consisted of 867768 events for V2V communications during three selected days, 
from which only the data related to the targeted agents was selected. The end matrix consisted of 120321 communication events for agent 52, 7063 for agent 38 and 127023 for agent 74 .

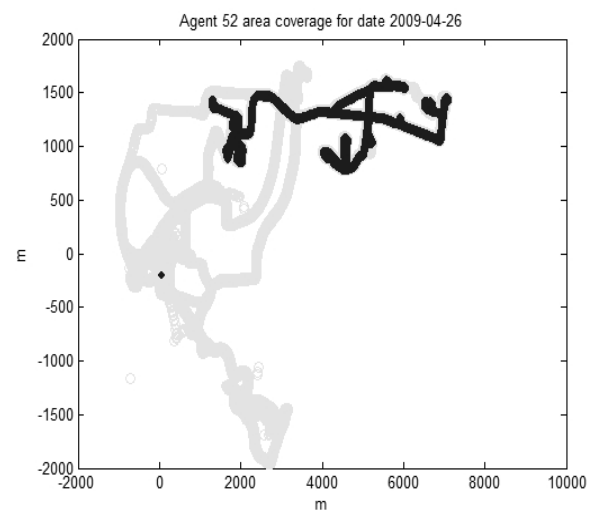

Fig. 2. Map of the route for agent 52 for the specified date

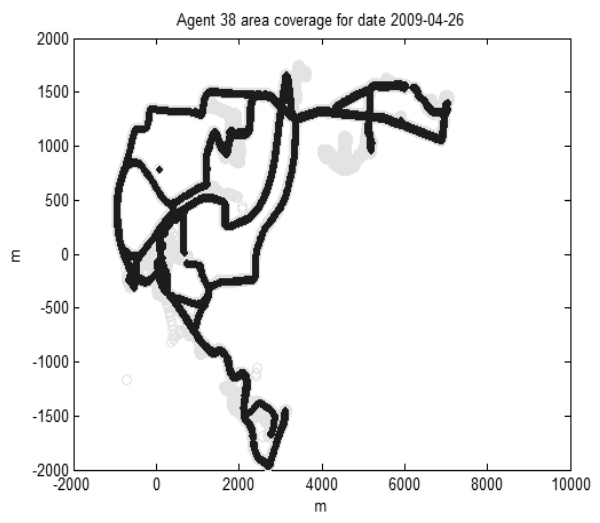

Fig. 3. Map of the route for agent 38 for the specified date

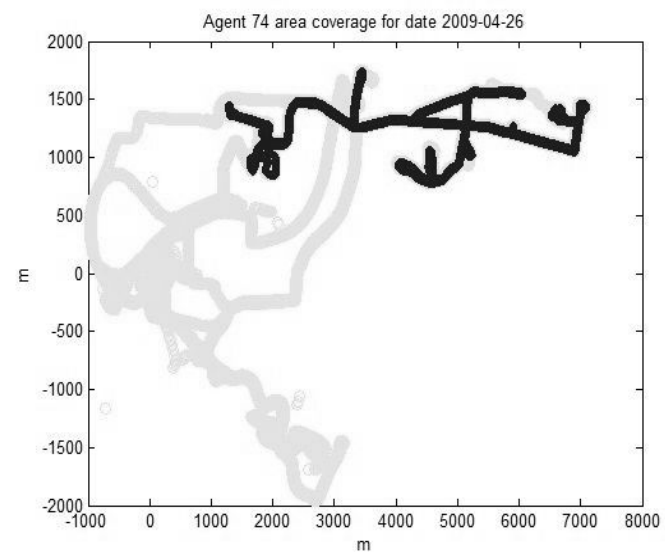

Fig. 4. Map of the route for agent 74 for the specified date

The collected data were divided into two sub-samples, train samples (75\%) and test/prediction samples (25\%). We conducted some experiments with different parameters of MLP using Levenberg-Marquardt (LM) learning algorithm. MLP structures were defined with two inputs, one or two hidden layers, with tansig or purelin activation functions and with a total number of neurons in the hidden layers $(10,20$ or 30). RBF networks were defined with 25 initial neurons and trained with different values of the Spread (S).

The output layer of the neural network has one neuron, which presents the vehicle speed. The input signals for the neural network training for each agent are presented in Figs 5, 6 and 7. The simulation results for each vehicle can be found in Tables 4, 5 and 6. Each table is formed by individual output results obtained from different MLP and RBF structures for selected agents 52, 38 and 74. 

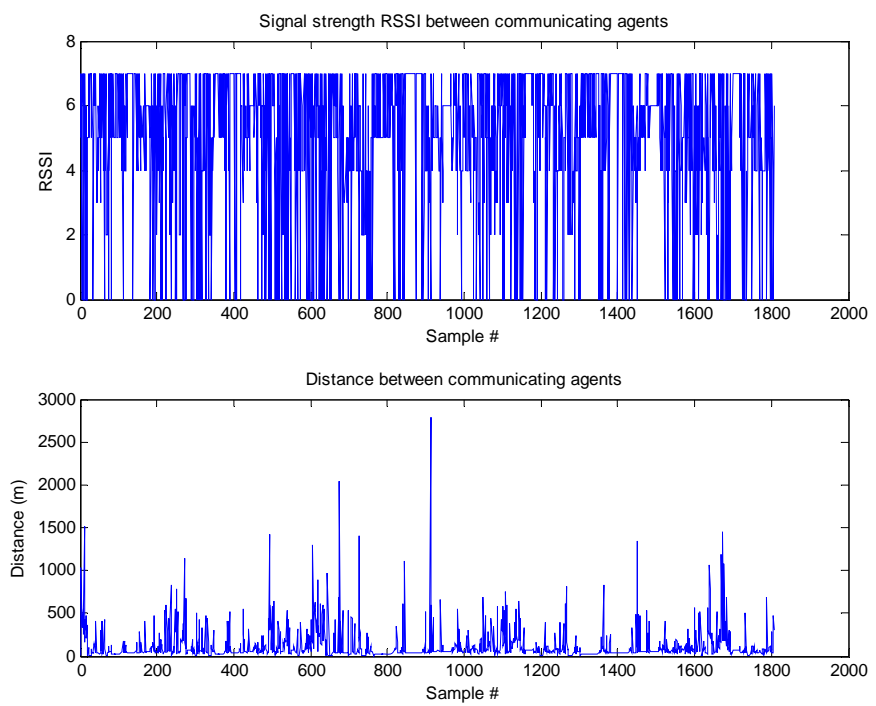

Fig. 5. Input data (signal strength and distance) for training (Agent 52)

Table 4. Results of the error for different neural network training for Agent 52

\begin{tabular}{|c|c|c|c|c|c|c|c|}
\hline \multirow{2}{*}{$\begin{array}{c}\text { NN } \\
\text { Type }\end{array}$} & NN & LR & Spread & MNE & $\begin{array}{c}\text { Performance } \\
\text { (MSE) }\end{array}$ & \multicolumn{2}{|c|}{ Output error predict } \\
\cline { 7 - 9 } & & & & & & $R^{2}$ & RMSE \\
\hline MLP & 10 & 0.001 & n/a & 500 & 6.26 & 0.2762 & 2.6350 \\
\hline MLP & 20 & 0.001 & n/a & 500 & 6.22 & 0.2730 & 2.3686 \\
\hline MLP & 30 & 0.001 & n/a & 1000 & 6.20 & 0.2851 & 2.3488 \\
\hline MLP & 10 & 0.01 & n/a & 500 & 6.35 & 0.2584 & 2.3923 \\
\hline MLP & 20 & 0.01 & n/a & 1000 & 6.21 & 0.2920 & 2.3375 \\
\hline MLP & 30 & 0.01 & n/a & 1000 & 6.13 & 0.2918 & 2.3378 \\
\hline RBF & 25 & n/a & 0.5 & 500 & 0.675 & 0.9053 & 0.8550 \\
\hline RBF & 25 & n/a & 0.1 & 500 & 0.087 & 0.9917 & 0.253 \\
\hline RBF & 25 & n/a & 0.05 & 500 & 0.029 & 0.9981 & 0.1211 \\
\hline
\end{tabular}
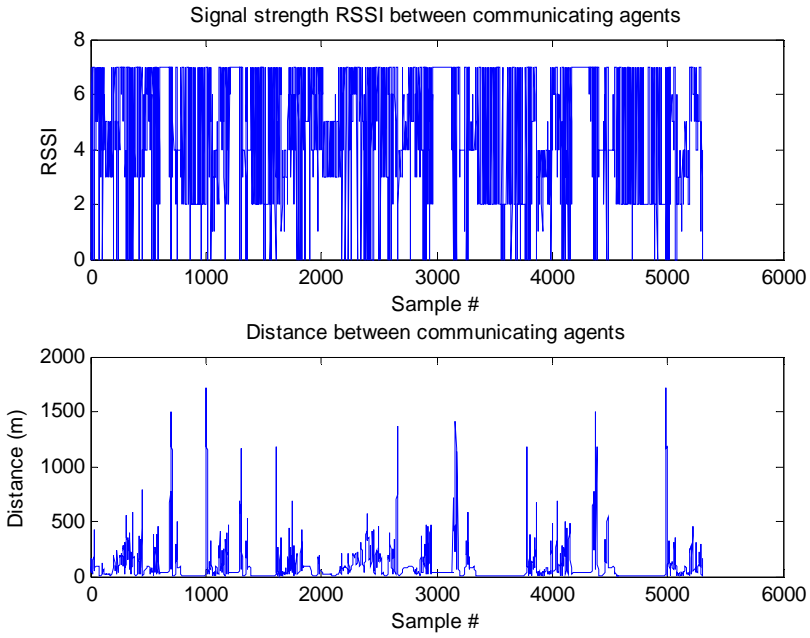

Fig. 6. Input data (signal strength and distance) for training (Agent 38) 
Table 5. Results of the error for different neural network training for Agent 38

\begin{tabular}{|c|c|c|c|c|c|c|c|}
\hline \multirow{2}{*}{$\begin{array}{c}\text { NN } \\
\text { Type }\end{array}$} & NN & LR & Spread & MNE & $\begin{array}{c}\text { Performance } \\
\text { (MSE) }\end{array}$ & \multicolumn{2}{|c|}{ Output error predict } \\
\cline { 7 - 9 } & & & & & $R^{2}$ & RMSE \\
\hline MLP & 10 & 0.001 & n/a & 500 & 12.7 & 0.5035 & 3.4311 \\
\hline MLP & 20 & 0.001 & n/a & 500 & 8.56 & 0.6872 & 2.7622 \\
\hline MLP & 30 & 0.001 & n/a & 500 & 10.4 & 0.6160 & 3.0175 \\
\hline MLP & 10 & 0.01 & n/a & 500 & 14.2 & 0.4072 & 3.7493 \\
\hline MLP & 20 & 0.01 & n/a & 500 & 6.52 & 0.6245 & 2.9838 \\
\hline MLP & 30 & 0.01 & n/a & 1000 & 8.37 & 0.6687 & 2.8026 \\
\hline RBF & 25 & n/a & 0.5 & 500 & 0.11 & 0.9962 & 0.3005 \\
\hline RBF & 25 & n/a & 0.1 & 500 & 0.065 & 0.9979 & 0.2218 \\
\hline RBF & 25 & n/a & 0.05 & 500 & 0.065 & 0.9979 & 0.2218 \\
\hline
\end{tabular}
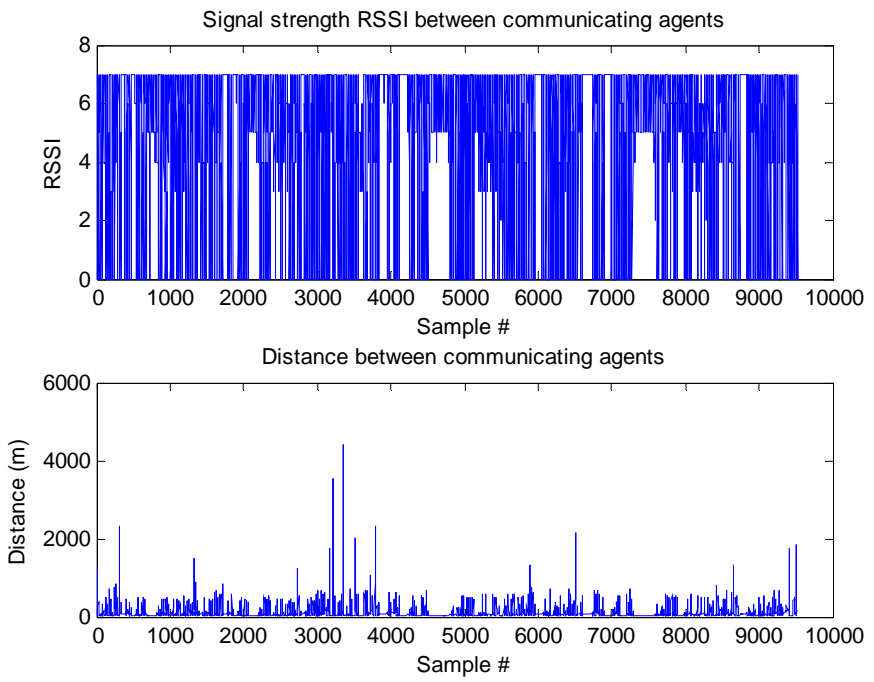

Fig. 7. Input data (signal strength and distance) for training of agent 74

Table 6. Results of the error for different neural network training for Agent 74

\begin{tabular}{|c|c|c|c|c|c|c|c|}
\hline $\begin{array}{c}\text { NN } \\
\text { Type }\end{array}$ & NN & LR & Spread & MNE & $\begin{array}{l}\text { Performance } \\
\text { (MSE) }\end{array}$ & \multicolumn{2}{|c|}{ Output error predict } \\
\cline { 6 - 8 } & & & & & & $R^{2}$ & RMSE \\
\hline MLP & 10 & 0.001 & n/a & 500 & 5.43 & 0.2482 & 2.1992 \\
\hline MLP & 20 & 0.001 & n/a & 500 & 5.50 & 0.2394 & 2.2120 \\
\hline MLP & 30 & 0.001 & n/a & 1000 & 5.36 & 0.2357 & 2.2173 \\
\hline MLP & 10 & 0.01 & n/a & 500 & 5.31 & 0.2436 & 2.2059 \\
\hline MLP & 20 & 0.01 & n/a & 1000 & 5.37 & 0.2563 & 2.1873 \\
\hline MLP & 30 & 0.01 & n/a & 1000 & 5.38 & 0.2516 & 2.1941 \\
\hline RBF & 25 & n/a & 0.5 & 500 & 0.2 & 0.9868 & 0.3345 \\
\hline RBF & 25 & n/a & 0.1 & 500 & 0.034 & 0.9997 & 0.0436 \\
\hline RBF & 25 & n/a & 0.05 & 500 & 0.001 & 1.000 & 0.0108 \\
\hline
\end{tabular}

During the neural network training for MLP networks we used a fixed number of 2 hidden layers, tansig activation function in hidden and purelin for output layers, 
and changed the Number of Neurons in hidden layers $(N N)$, the maximum number of epochs $(M N E)$ and the Learning Rate $(L R)$. We recorded the targets performances.

During the neural network training for RBF networks with 25 initial neurons, the target performance after 500 epochs was calculated. Three training attempts were made while the Spread (S) parameter was modified.

For each neural network configuration we calculated the Root Mean Square Error (RMSE), Equation (2) and the coefficient of determination $\left(R^{2}\right)$. Equation (3) for the parameters between the real vehicle speeds and values from the neural network learning of the prediction set.

$$
\begin{gathered}
\text { RMSE }=\sqrt{\frac{1}{n} \sum_{i=1}^{N}\left(y_{i}-f_{i}\right)^{2}}, \\
R^{2} \equiv 1-\frac{S S_{\text {res }}}{S S_{\text {tot }}} ; S S_{\text {res }}=\sum_{i}\left(y_{i}-f_{i}\right)^{2} ; S S_{\text {res }}=\sum_{i}\left(y_{i}-\bar{y}_{i}\right)^{2},
\end{gathered}
$$

where $y_{i}$ and $f_{i}$ are the experimental and forecasted values, respectively and $n$ is the total number of the test data.

According to the results in Tables 4, 5 and 6, the best network configurations with minimum values of RMSE and $R^{2}$, achieved by the trained MLP and RBF network configurations for each of the three agents are presented in Table 7.

\begin{tabular}{|c|c|c|c|c|c|c|c|c|}
\hline \multirow{2}{*}{ Agent } & \multicolumn{2}{|c|}{$\begin{array}{c}\text { Number } \\
\text { of analyzed samples }\end{array}$} & \multicolumn{3}{|c|}{$\begin{array}{l}\text { MLP with the best } \\
\text { training performance }\end{array}$} & \multicolumn{3}{|c|}{$\begin{array}{l}\text { RBF with the best } \\
\text { training performance }\end{array}$} \\
\hline & Training & Prediction & $R^{2}$ & RMSE & Parameters & $R^{2}$ & RMSE & Parameters \\
\hline Agent 54 & 1805 & 601 & 0.2920 & 2.3375 & $\begin{array}{c}\mathrm{NN}=20 \\
\mathrm{LR}=0.001 \\
\mathrm{MNE}=1000\end{array}$ & 0.9981 & 0.1211 & $\begin{array}{c}S=0.05 \\
\mathrm{MNE}=500\end{array}$ \\
\hline Agent 38 & 1325 & 447 & 0.6872 & 2.7622 & $\begin{array}{c}\mathrm{NN}=20 \\
\mathrm{LR}=0.01 \\
\mathrm{MNE}=1000\end{array}$ & 0.9979 & 0.2218 & $\begin{array}{c}S=0.05 \\
M N E=500\end{array}$ \\
\hline Agent 74 & 1465 & 488 & 0.2516 & 2.1941 & $\begin{array}{c}\mathrm{NN}=20 \\
\mathrm{LR}=0.01 \\
\mathrm{MNE}=1000\end{array}$ & 1 & 0,0108 & $\begin{array}{c}S=0.05 \\
\mathrm{MNE}=500\end{array}$ \\
\hline
\end{tabular}

Table 7. The best neural network parameters for the selected three agents

ANN output results have shown very accurate prediction of the new samples. The results were compared in terms of their training effort and network performance when encountering new data. MLP structures for each vehicle agent, with two hidden layers with 20 neurons in the hidden layers, trained through 1000 epochs, produced the best combination of accuracy and a low cost of training. RBF structures for each vehicle agent, with a spread value of 0.05 trained through 500 epochs, have given the best results.

Figs 8, 9 and 10 show examples of the comparison between the best performance results obtained from the trained ANN models using MLP and RBF neural network structures for each vehicle agent 52, 38 and 74, respectively. 

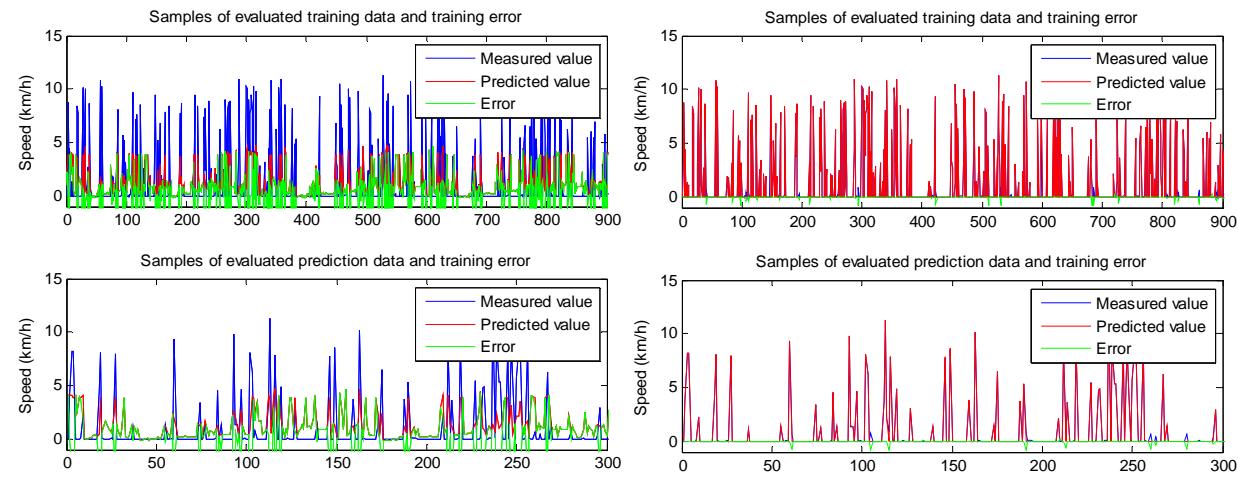

(a)

(b)

Fig. 8. The best performance for agent 52 data using: MLP NN (a); RBF NN (b)
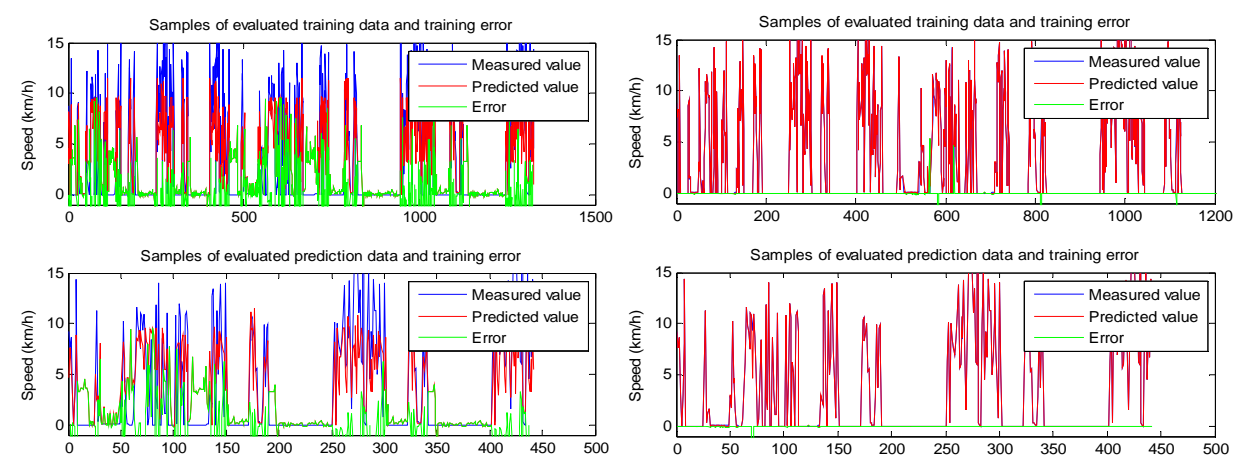

(a)

(b)

Fig. 9. The best performance for agent 38 data using: MLP NN (a); RBF NN (b)
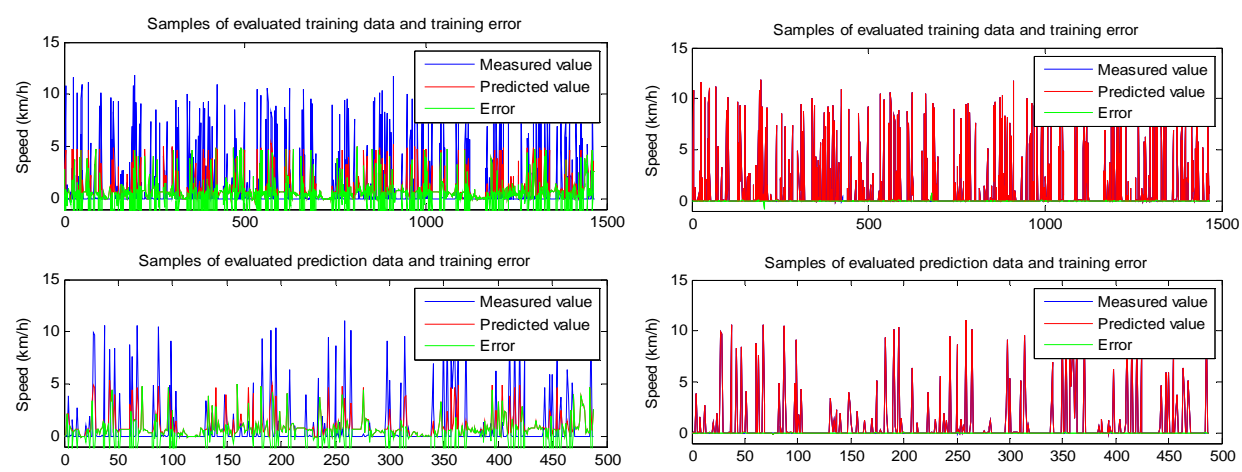

(a)

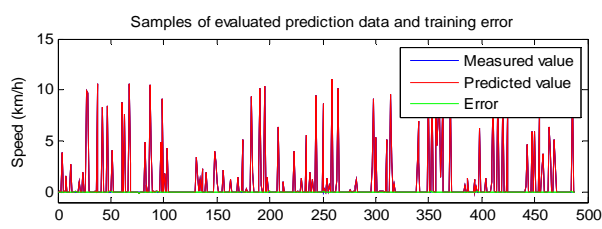

(b)

Fig. 10. The best performance for agent 74 data using: MLP NN (a); RBF NN (b)

\section{Conclusion and future work}

We have presented an intelligent transport system based on the multi-vehicle interaction prediction analysis of the traffic speed. In this paper we analyze a few V2V interactions, each of them having its own evaluation agent. In order to test the prediction feasibility of the proposed multiagent intelligent systems, a case study of 
a large data set is used and discussed. A new prediction speed approach (V2VSPP) was developed using an extensive data set from the Australian Centre for Field Robotics. It contains vehicle state information, vehicle-to-vehicle communications and road maps of high temporal resolution for large numbers of interacting vehicles over a long time period (3 year). The forecasting is made based on the distance between the vehicles and the Received Signal Strength Indicator (RSSI) values for communications between pairs of vehicles. The V2VSPP can predict the traffic speed profile for each vehicle agent.

The significance of this is that such an ANN can enable extensive parametric studies to be carried out inexpensively, in order to predict the speed profiles based on V2V cooperative interactions in multi-vehicle trajectories setup. At the next stage, we will test the system proposed with a large number of vehicle agents in order to help the operators in the traffic centers to identify the optimal global control action.

Acknowledgements: The paper is partially supported by TUD COST TU 1102 "ARTS - Towards Autonomic Road Transport Support Systems” and FP7 Project 316087 ACOMIN “Advance Computing and Innovation”.

\section{References}

1. Ch e n, L.-W., Y.-H. P en g, Y.-C. T s en g. An Infrastructure-Less Framework for Preventing Rear-End Collisions by Vehicular Sensor Networks. - IEEE Commun. Lett., Vol. 15, 2011, No 3, pp. 358-360.

2. Controller Area Network (CAN-Bus). http://www.gaw.ru/data/Interface/CAN_BUS.PDF

3. Ha fner, M., D. Cunn ingham, L. Caminiti, D. D. Ve c chi o. Cooperative Collision Avoidance at Intersections: Algorithms and Experiments. - IEEE Trans. Intell. Transp. Syst., Vol. 14, 2013, No 3, pp. 1162-1175.

4. D i a, H. An Object-Oriented Neural Network Approach to Short-Term Traffic Forecasting. - Eur. J. Oper. Res., Vol. 131, 2001, No 2, pp. 253-261.

5. IEEE 1609-Family of Standards for Wireless Access in Vehicular Environments (WAVE). http://vii.path.berkeley.edu/1609_wave/

6. K a t s a r g y r i, G.-E., I. V. K o l m a n o v s k y, J. M i c h e l i n i. Optimally Controlling Hybrid Electric Vehicles Using Path Forecasting. - In: Proc. of Amer. Control Conf., 2009, pp. 4613-4617.

7. L e e, E., J. K i m, W. Y o o n. Traffic Speed Prediction under Weekday, Time, and Neighboring Links' Speed: Back Propagation Neural Network Approach. - In: Lecture Notes in Computer Science. Berlin, Germany, Springer-Verlag, 2007, pp. 626-635.

8. L e vin s o n, J., J. A sk e l a nd, J. B e c ker, J. D o l s o n, D. H eld, S. K a m m el, J. Ko l t e r, D. Langer, O. Pink, V. Pratt, M. Sokolsky, G. Stanek, D. S tavens, A. T e i c h m a n, M. W e r ling, S. Th r u n. Towards Fully Autonomous Driving: Systems and Algorithms. - In: Proc. of IEEE Intelligent Vehicles Symposium (IV), 2011, pp. 163-168.

9. Milanés, V., J. P erez, E. Onieva, C. G onzalez. Controller for Urban Intersections Based on Wireless Communications and Fuzzy Logic. - IEEE Trans. Intell. Transp. Syst., Vol. 11, 2010, No 1, pp. 243-248.

10. N a s i m, R., A. Ka s s le r. Distributed Architectures for Intelligent Transport Systems: A Survey. - In: Proc. of 12th IEEE 2nd Symposium on Network Cloud Computing and Applications, 2012.

11. On-Board Diagnostics (OBD). http://www.epa.gov/otaq/regs/im/obd/index.htm 
12. P a r k, J., Y. L. M u r phe y, J. G. Kr i st in s s on, R. M c G e e, M. L. K u ang, T. Phillips. Real Time Vehicle Speed Prediction Using a Neural Network Traffic Model. - In: Proc. of IEEE IJCNN, 2011, pp. 2991-2996.

13. Park, J., Y. L. Murphey, R. Mc Gee, J. G. Kristins on, M. L. Kuang, A. M. $\mathrm{P}$ h i ll i p s. Intelligent Trip Modeling for the Prediction of an Origin-Destination Traveling Speed Profile. - IEEE Transactions on Intelligent Transportation Systems, Vol. 15, 2014.

14. Ploeg, J., S. Shladover, H. Nijmeijer, N. Van de Wouw. Introduction to the Special Issue on the 2011 Grand Cooperative Driving Challenge. - IEEE Trans. Intell. Transp. Syst., Vol. 13, 2012, No 3, pp. 989-993.

15. Q i u, W., L. Z h a n g. Integration of Cyber Physical System Based on Aspect Oriented. - JCIT, Vol. 7, 2012, No 22, pp. 368- 375.

16. Q u re s h i, K. N., A. H. A b d u l l a h. A Survey on Intelligent Transportation Systems. - MiddleEast Journal of Scientific Research, Vol. 15, 2013, No 5, pp. 629-642.

17. R a u c h, A., S. M a i e r, F. K l a n n e r, K. D i e t m a y e r. Inter-Vehicle Object Association for Cooperative Perception Systems. - In: Proc. of 16th Int. IEEE Conf. Intelligent Transportation Systems, 2013, pp. 893-898.

18. S a d e k, A. W. Artificial Intelligence Applications in Transportation. Transport Research CIRCULAR, Number EC-113, 2007.

19. S c hulz e, M., J. Z. R i ve ro s. Impact of Electrical Vehicles on Strategic Planning of Energy Infrastructure. - In: Proc. of Int. Conf. POWERCON, 24-28 October 2010, pp. 1-6.

20. Sang, C., U. Suh, J. Tanik, J. N. Carbone, A. Eroglu. (Eds) Applied Cyber-Physical Systems. Springer, 2014.

21. Sivaraman, S., M. M. Trivedi. Towards Cooperative, Predictive Driver Assistance. - In: Proc. of 16th Int. IEEE Conf. Intelligent Transportation Systems, 2013, pp. 1719-1724.

22. S o n g, Z., Y. Q. C h e n, C. R. S a stry, N. C. T a s. Optimal Observation for Cyber-Physical Systems. Springer, 2009.

23. S o t e l o, M., J. W. C. V a n L in t, U. N un es, L. V la c i c, M. Ch ow dhu ry. Introduction to the Special Issue on Emergent Cooperative Technologies in Intelligent Transportation Systems. - IEEE Trans. Intell. Transp. Syst., Vol. 13, 2012, No 1, pp. 1-5.

24. Tacconi, D., et al. Using Wireless Sensor System Networks to Support Intelligent Transportation Systems. - Ad Hoc Networks, Vol. 8, 2010, No 5, pp. 462-473.

25. Trivedi, P., K. Deshmukh, M. Shrivastava. Cloud Computing for Intelligent Transportation System. - International Journal of Soft Computing and Engineering (IJSCE), Vol. 2, 2012, Issue 3, ISSN: 2231-2307.

26. Unbehauen, H. Identification of Nonlinear Systems, Control Systems. - Robotics and Automation. Vol. VI. Identification of Nonlinear Systems - Encyclopedia Of Life Support Systems (EOLSS), 2009.

27. Verdone, R., et al. Wireless Sensor and Actuator Networks: Technologies, Analysis and Design. -Academic Press. Ad Hoc Networks, Vol. 8, 2010, No 5, pp. 462-473.

28. W a r d, J., S. W or r a l l, G. A g a m e n n o n i, E. N e b o t. The Warrigal Dataset: Multi-Vehicle Trajectories and V2V Communications. - IEEE Intelligent Transportation Systems Magazine, Vol. 109, 2014.

29. Worrall, S., G. Agamennoni, J. Nieto, E. Nebot. A Context-Based Approach to Vehicle Behavior Prediction. - IEEE Intell. Transp. Syst. Mag., Vol. 4, 2012, No 3, pp. 32-44.

30. W u a, F.-J., Y.-F. Ka ob, Y.-C. Ts eng. From Wireless Sensor Networks Towards Cyber Physical Systems. - Pervasive and Mobile Computing, Vol. 7, 2011, Vol. 9, 2009, No 11, pp. 8824-8830.

31. Y a n g, X., M. B e hr o o z i, O. A. O l a t u n b o s u n. A Neural Network Approach to Predicting Car Tyre Micro-Scale and Macro-Scale Behaviour. - Journal of Intelligent Learning Systems and Applications, 2014, No 6, pp. 11-20.

32. Y e, F., M. A d a m s, S. R o y. V2V Wireless Communication Protocol for Rear-End Collision Avoidance on Highways. - In: Int’l Conf. Communications, 2008, pp. 375-379. 